\title{
A EFICIÊNCIA DOS GASTOS MUNICIPAIS EM EDUCAÇÃO NO ESTADO DO PARANÁ
}

\section{EFFICIENCY OF MUNICIPAL SPENDING ON EDUCATION IN THE STATE OF PARANÁ}

\author{
Wanderson Dutra Gresele* \\ Eliana Cunico ${ }^{* *}$
}

Resumo: Este estudo objetiva avaliar a eficiência dos gastos públicos no ensino básico, especificamente para os anos iniciais do ensino fundamental, nos municípios do Estado do Paraná no ano de 2017. Foi utilizada a Análise Envoltória de Dados (DEA), que consiste em um método de programação matemática, capaz de medir as eficiências técnica, de escala e total para a relação produto/insumo. A pesquisa é classificada como quantitativa, descritiva de resultados e os softwares $R$, SPSS e QGIS foram utilizados na análise de resultados. Os resultados indicam que não necessariamente uma maior alocação de recursos proporcionará melhor desempenho no IDEB, diante de uma aplicação ineficiente. Ademais, o nível médio de eficiência técnica dos municípios é de $58,5 \%$, com um desvio padrão de 17,5 ; isso significa que os municípios podem, em média, reduzir $41,5 \%$ dos insumos sem comprometer seus resultados. $\mathrm{Na}$ análise da eficiência da relação outputs/inputs (insumos/produto) na educação dos municípios paranaenses por meio das possiveis combinações dos tipos de retornos e, com base nos dados, conclui que mudanças nas práticas de gestão se fazem necessárias, dado que o aporte de novos recursos financeiros não irá impactar o desempenho educacional da maioria dos municípios.

\footnotetext{
* Mestrado em Administração pela Universidade Federal do Paraná (2011)

** Doutora em Administração pela Universidade Estadual de Maringá; Docente no Curso de Administração da Universidade Estadual do Oeste do Paraná - UEM/PR, Campus de Marechal Cândido Rondon.
} 
Palavras-Chave: Análise Envoltória de Dados; DEA; recursos públicos.

\begin{abstract}
This study aims to assess the efficiency of public spending on basic education, specifically the early years of elementary school, in the municipalities of the State of Paraná in 2017. Data Envelopment Analysis (DEA) was used, which consists of a mathematical programming method, capable of measuring efficiencies technical, scale and total, for the product/input ratio. The research is classified as quantitative, descriptive and uses Data Envelopment Analysis (DEA). The software R, SPSS and QGIS were used in the analysis of results. The indicate that not necessarily a greater allocation of resources will provide better IDEB results, in the face of an inefficient application. In addition, the average level of technical efficiency in the municipalities is $58.5 \%$, with a standard deviation of 17.5 ; this means that municipalities can, on average, reduce $41.5 \%$ of inputs without compromising their results. The work analyzes the efficiency of the outputs/inputs ratio in the education of the municipalities of Paraná through the possible combinations of the types of returns and, based on the data, concludes that changes in management practices are necessary, given that the contribution new financial resources will not impact the educational performance of most municipalities.
\end{abstract}

Keywords: Data Envelopment Analysis; DEA; public resources.

Resumen: Este estudio objetiva evaluar la eficiencia del gasto público en educación básica, específicamente los primeros años de la escuela primaria, en los municipios del Estado de Paraná en 2017. Se utilizó el Análisis Envolvente de Datos (DEA), el cual consiste en un método de programación matemática, capaz de medir eficiencias, técnicas, escala y total, para la relación producto/insumo. La investigación se clasifica como cuantitativa, descriptiva de resultados y en el análisis de resultados se utilizó el software R, SPSS y QGIS. Los resultados indican que no necesariamente una mayor asignación de recursos proporcionará mejores resultados en IDEB, ante una aplicación ineficiente. Además, el nivel promedio de eficiencia técnica en los municipios es de $58,5 \%$, con una desviación estándar de 17,5; esto significa que los municipios pueden, en promedio, reducir el 41,5\% de los insumos sin comprometer sus resultados. En el análisis de la eficiencia de la relación de productos / insumos en educación en los municipios de Paraná a través de combinaciones de tipos de retornos y, con base en los datos, se concluye que los cambios en las prácticas de gestión son obligatorios, dado que el aporte de nuevas Los recursos no afectarán el rendimiento educativo de la mayoría de los municipios.

Palabras Clave: Análisis envolvente de datos; DEA; recursos públicos. 
GRESELE, W. D.; CUNICO, E. A eficiência dos gastos municipais em educação...

\section{Introdução}

Não é de agora que o Brasil tem enfrentado desafios na gestão pública, agravada pela recessão econômica e crise sanitária da COVID-19, fatores que reduzem o investimento em politicas públicas e aumentam a demanda por serviços básicos, principalmente pela queda na arrecadação do estado e da massa salarial (MONTE; LEOPOLDINO, 2017). É fato considerar que a qualidade dos serviços públicos não é diretamente relacionada à quantidade de dinheiro aportado, ou seja, o problema da escassez dos recursos pode ser minimizado com a utilização eficiente, para conseguir resultados melhores com os meios disponiveis, o que indica um desafio gerencial nos gastos públicos do Brasil (DINIZ; CORRAR, 2011; MATTOS; TERRA, 2015a; SILVA; MORETTI; SCHUSTER, 2016; LEOPOLDINO, 2017; MATIAS et al., 2018; ZUBYK et al., 2019).

A utilização eficiente do gasto público em educação tem impactos significativos no desenvolvimento econômico por proporcionar um aumento no nível de capital humano (SOUZA; RUTALIRA, 2016), ou ainda, pela escolarização formal ser chave para o desenvolvimento econômico e social, motivos pelos quais o Brasil investe de forma contínua nesta área (SAVIAN; BEZERRA, 2013).

Dado a importância econômico-social e, notadamente, a publicização dos dados que possibilitam mensurar o desempenho das escolas públicas, tal como o Índice de Desenvolvimento da Educação Básica (IDEB), recebe destaque no Brasil a discussão sobre a eficiência do sistema educacional, principalmente nas escolas públicas. É possivel em um primeiro momento pensar que o aumento significativo de recursos para a educação seria a solução, mas isso, por si só, pode não garantir melhoria educacional (DINIZ; CORRAR, 2011).

Em média, o total de recursos para educação em mãos dos governos municipais aumentou nos últimos anos (ROCHA, 2013), e irá ter novos incrementos como o Novo Fundeb (Fundo de Manutenção e Desenvolvimento do Ensino Básico e de Valorização dos Profissionais da Educação) (BRASIL, 2020). Entretanto os resultados do Programa Internacional de Avaliação de Estudantes (PISA), apresentem que o cenário nacional indica defasagem dos alunos nos parâmetros de desempenho escolar (PEÑA, 2012; CASTRO; SOUSA, 2018). 
Ao pensar a área de ensino com um sistema que se utiliza de recursos na busca de resultados, há que se considerar que esse é um processo que combina alunos, características das escolas, fatores institucionais e dinheiro para se ter uma educação de qualidade. Para tanto, torna-se latente analisar a eficiência no processo de alocação de recursos (inputs) na educação, por fornecer uma comparação dos desempenhos de unidades tomadoras de decisões, a fim de permitir melhor desempenho dos ineficientes, sem aumentar recursos empregados, apenas utilizando-os de forma mais eficiente (FERREIRA; GOMES, 2012; SAVIAN; BEZERRA, 2013).

A problemática desta pesquisa busca responder: Como se apresenta a eficiência relativa dos investimentos públicos no ensino fundamental nos municípios do Estado do Paraná no ano de 2017? Como forma de respondê-la, o trabalho objetiva analisar a eficiência relativa dos investimentos no ensino fundamental nos municípios paranaenses no ano de 2017. Para o alcance do objetivo proposto, o presente artigo utiliza-se da Análise Envoltória de Dados, também conhecida como DEA.

\section{Referencial Teórico}

\subsection{Políticas de avaliação na educação brasileira}

A Constituição da República de 1988 ordena que a educação é um direito social e uma obrigação do estado (BRASIL, 1988). Especificamente, o ordenamento determinou como responsabilidade dos municípios a atuação na educação infantil - prioritariamente - e no ensino fundamental, denominado de municipalização do ensino (ROCHA, 2013).

No ensino fundamental, um ponto importante para a educação brasileira é a Emenda Constitucional n ${ }^{\circ}$ 14, de setembro de 1996, que instituiu o Fundo de Manutenção e Desenvolvimento do Ensino Fundamental e de Valorização do Magistério (FUNDEF), o qual regulamentou o financiamento da educação pública fundamental brasileira. Outro ponto trata da instituição do Fundo de Manutenção e Desenvolvimento da Educação Básica e de Valorização dos Profissionais da Educação (FUN- 
DEB), que passou a vigorar em 2007 com o objetivo de contribuir para a universalização da educação básica, bem como promover a equidade, melhorar a qualidade do ensino e valorizar os profissionais da educação (SAVIAN; BEZERRA, 2013).

O Brasil, na década de 1990, passa a se preocupar com a criação de uma política nacional de avaliação, implantando o Sistema de Avaliação da Educação Básica (SAEB), o Exame Nacional do Ensino Médio (ENEM) e o Exame Nacional de Curso (ENC), conhecido como "Provão", para o ensino superior, substituído em 2004 pelo Exame Nacional de Desempenho de Estudantes (ENADE). Esses instrumentos permitiram a construção de uma valiosa base de dados para a elaboração de diagnósticos precisos sobre problemas relativos ao desempenho de estudantes e a política educacional em geral (VIDAL; VIEIRA, 2011).

No ano de 2020, a Emenda Constitucional 108/2020 traz o Novo Fundeb, que começa a vigorar a partir de 2021. O regulamento do novo programa, previsto então pelo novo artigo 212-A da Constituição Federal, se deu pela Lei 14.113/2020 de 25 de dezembro de 2020. Uma das maiores modificações é o aumento previsto na complementação dos recursos da União junto aos municipios e estados. A atual contribuição de $10 \%$ deve aumentar gradativamente até atingir 23\% dos recursos que formarão o fundo em 2026.

Outros índices foram criados na busca de acompanhar o desenvolvimento dos alunos, como é o caso do IDEB (Índice de Desenvolvimento da Educação Básica) em 2006, cuja função é sintetizar os resultados das avaliações desenvolvidas no sistema de ensino SAEB e Prova Brasil, para que possam ser assimilados mais facilmente, permitindo, assim, o estabelecimento de metas de qualidade educacional (INEP, 2018).

O IDEB apresenta-se como condutor de politicas públicas em prol da qualidade da educação, sendo uma ferramenta para acompanhamento das metas de qualidade do Plano de Desenvolvimento da Educação (PDE) para a educação básica, que tem estabelecido, como meta, que em 2022 o Brasil ultrapasse a nota 6 , média que corresponde a um sistema educacional de qualidade comparável a dos países desenvolvidos (INEP, 2018). 
Essas avaliações permitiram a construção de um padrão cujas variáveis pudessem ser desagregadas por sistemas de ensino e escolas. A implementação de um indicador de ordem geral por parte do Ministério da Educação permitiu tornar o debate em torno de resultados do processo educativo mais transparente e objetivo e, com isso, foi possível avaliar problemas de nossas politicas educacionais (VIDAL; VIEIRA, 2011).

\subsection{Análise da eficiência da educação por meio da DEA}

O processo de aprendizagem pode ser analisado sob a ótica da relação input/output, dado que combina vários recursos, tais como: alunos, características das escolas, fatores institucionais, dinheiro investido e os compara com os resultados. Têm-se assim parâmetros mensuráveis para avaliar a eficiência relativa, o qual padroniza os desempenhos, como no IDEB, pelos recursos empregados (ROCHA, 2013).

Estudos que analisam o desempenho das unidades educacionais por meio da DEA (Análise Envoltória de Dados), têm sido amplamente utilizados na avaliação da eficiência no uso de recursos públicos em educação (LOURENÇO et al., 2017; CASTRO; MONTE; LEOPOLDINO, 2017; GRESELE; KRUKOSKI, 2018; MATIAS et al., 2018; SOUZA, 2018; ZUBIK et al., 2019).

Especificamente, no que tange ao Estado do Paraná, quatro estudos foram encontrados. Dentre eles, o estudo de Bohrer, Comunelo e Godarth (2013) investigou a eficiência do gasto público com a educação das 42 cidades do Sudoeste do Paraná em 2009, utilizando como input o custo por aluno e como outputs média nas disciplinas de Português e Matemática na Prova Brasil. Curiosamente, os municípios que possuíam os maiores custos por aluno, não figuram nas primeiras posições da eficiência na gestão dos gastos com educação.

O estudo de Savian e Bezerra (2013) avaliou a eficiência dos gastos públicos com a educação nas séries iniciais do ensino fundamental no Paraná, nos anos de 2005 e 2009, com a DEA. O estudo dos autores abrangeu 381 municípios do Estado do Paraná. Utilizaram gasto por 
aluno; número de escolas; relação aluno/professor e PIB per capita municipal como inputs e a nota do IDEB municipal como output. Em resumo, a maioria dos municípios se mostraram ineficientes, o que demonstra a necessidade de revisão quanto aos meios de alocação dos recursos.

Gresele e Krukoski (2018) avaliaram a eficiência dos gastos públicos no ensino fundamental nos municípios paranaenses no ano de 2015, também pela técnica DEA. Como input foram utilizados gasto por aluno, docentes por alunos e escolas por alunos e como output a nota do IDEB do municipio. Os autores identificaram quatro grupos de municípios, sendo que alguns alcançaram resultados melhores com menores aportes de recursos e outros que, mesmo realizando um significativo aporte de recursos, não obtiveram bons resultados.

Recentemente, o estudo de Zubyk et al. (2019) realizou uma análise da eficiência dos gastos dos municípios paranaenses em saúde e educação, por meio da técnica DEA, para os anos de 2001 a 2012. Os gastos com educação Per Capita, Gastos com Saúde Per Capita foram utilizados como inputs e o Índice IPARDES (Instituto Paranaense de Desenvolvimento Econômico e Social) de Desenvolvimento Municipal (IPDM)de Educação e de Saúde como output. O estudo relevou que aproximadamente $8 \%$ dos municípios apresentaram eficiência máxima, além do fato de que os municípios que atingiram altos niveis de eficiência, não conseguiram manter-se na posição dos anos posteriores.

\section{Metodologia}

Esta pesquisa configura-se como quantitativa e descritiva em relação a seu objeto, uma vez que há relação entre variáveis. Para analisar a eficiência dos investimentos públicos no ensino fundamental dos municipios paranaenses no ano de 2017 por meio da técnica DEA., foi escolhido o modelo BCC, a fim de comparar unidades tomadoras de decisões com portes diferentes. Quanto à orientação do modelo, o presente estudo utilizou-se de uma orientação para os insumos (inputs).

Como output, o índice escolhido foi o IDEB do município e, como input, os gastos por aluno, docentes por alunos e escolas por alunos (SA- 
VIAN; BEZERRA, 2013; LOURENÇO ET AL., 2017; MONTE; LEOPOLDINO, 2017; BEGNINI; TOSTA, 2017; CASTRO; SOUSA, 2018). Após, seguiu-se o pressuposto de que a seleção das variáveis deve obedecer ao princípio de máxima relação causal entre inputs e outputs, então, o método optado foi I-O Stepwise Exaustivo Completo, conforme propõe Senra, Mello e Meza (2007).

Dos 399 municípios paranaenses, 10 não fizeram parte do escopo final por falta de algum dado para o modelo, sendo: Adrianópolis, Colombo, Morretes, Nova América da Colina, Porto Rico, Presidente Castelo Branco, Rancho Alegre, Santa Cruz de Monte Castelo, São José da Boa Vista e Virmond.

A pesquisa documental ocorreu nas seguintes fontes de dados: Declarações de Contas Anuais, obtidos no site do Sistema de Informações Contábeis e Fiscais do Setor Público Brasileiro (SICONFI), da Secretaria do Tesouro Nacional, referente ao exercício de 2017; nos dados demográficos do Ensino Fundamental nos Anos Iniciais das Escolas Municipais do Estado do Paraná, por meio do Censo do Ensino Fundamental do ano de 2017, desenvolvido pelo Ministério da Educação; e nos índices do IDEB dos municípios.

Nas análises foram utilizados os softwares R, SPSS e QGIS. Para a Análise Envoltória de Dados, utilizou-se o software R, livre e open source. Destaca-se o pacote Benchmarking, como sendo o principal recurso computacional utilizado neste trabalho. A DEA foi utilizada para analisar a eficiência total, a eficiência técnica e a eficiência de escala dos municípios (BOGETOFT; OTTO, 2011). Na sequência, com os escores das eficiências, os inputs e o output, utilizou-se o software SPSS, com o objetivo de desenvolver estatísticas descritivas para as variáveis em estudo (FIELD, 2009; DANCEY; REIDY, 2013).

O QGis é um software livre e open source, multiplataforma de sistema de informação geográfica que permite a visualização, edição e análise de dados georreferenciados, foi utilizado para uma melhor representação dos dados, utilizando-se das coordenadas vetoriais disponíveis pelo Instituto Brasileiro de Geografia (IBGE, 2018). Foram desenvolvidos mapas georreferenciados de desempenho apresentados nos resultados a seguir. 
GRESELE, W. D.; CUNICO, E. A eficiência dos gastos municipais em educação...

\subsection{Análise Envoltória de Dados}

O desenvolvimento prático da técnica de Análise Envoltória de Dados se deu em 1978 com a tese de doutorado de Edwardo Rhodes, com a orientação de Willian Wager Cooper. Cooper então utilizou o método de programação matemática, partindo do conceito de eficiência técnica para a relação produto/insumo desenvolvido por Farrel no ano de 1957, para a construção de um modelo para comparar o desempenho das escolas (FERREIRA; GOMES, 2012; MATTOS; TERRA, 2015b).

O modelo inicial do DEA, desenvolvido pelos autores Charnes, Cooper e Rhodes, denominado de CCR, pressupõe retornos constantes de escala, isso quer dizer que uma variação nos inputs produz uma mudança equiproporcional nos outputs. De outra maneira, um aumento de $\mathrm{k} \%$ na utilização de todos os insumos aumenta os resultados na mesma proporção de k\% (BOUERI, 2015; MATTOS; TERRA, 2015b).

Sob o olhar do modelo CCR apenas a DMU C (unidade tomadora de decisão, em tradução livre) é considerada eficiente. Assim, para estimar a ineficiência das DMU's que estão fora da fronteira de eficiência $\left(\mathrm{FE}_{\mathrm{RCE}}\right)$ o modelo CCR efetua uma projeção radial, como pode ser visualizado no movimento dos pontos G-Gb da Figura 1.

Figura 1- Fronteiras de Eficiência BCC e CCR

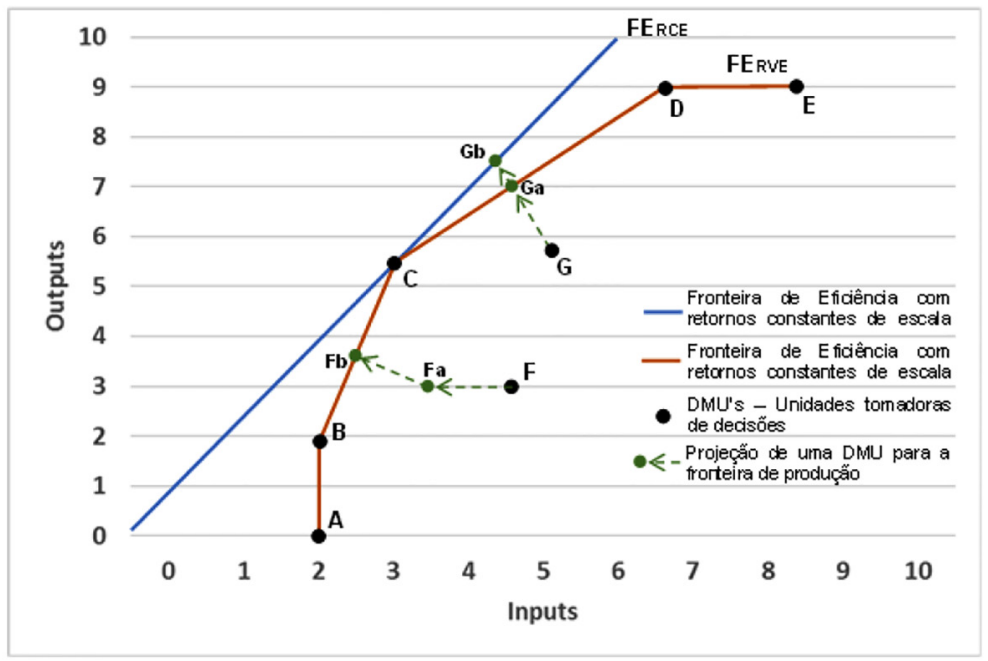

Fonte: Adaptado de Cooper et al. (2006, p. 116). 
Entretanto, é possivel que uma função de produção apresente outros tipos de retornos de escala além do constante. Isso significa que a depender da quantidade de insumos empregada para fins de produção uma DMU pode operar em retornos crescentes, decrescentes ou constantes. Se um aumento de $\mathrm{k} \%$ na utilização de todos os insumos aumenta o produto em mais do que $\mathrm{k} \%$, dizemos que a função de produção apresenta retornos crescentes à escala. Entretanto, se esse mesmo aumento produzir uma variação no produto menor do que $\mathrm{k} \%$, dizemos que a tecnologia de produção apresenta retornos decrescentes à escala. (MATTOS; TERRA, 2015b). Na Figura 1, o trecho A-C (exclusive) apresenta retornos crescentes de escala; o ponto $\mathrm{C}$ apresenta o único trecho com rendimentos constantes de escala; e o trecho representado pelos pontos $\mathrm{C}$ (exclusive)-E apresentam rendimentos decrescentes.

Assim, dado que algumas unidades tomadoras de decisões podem estar operando com retornos variáveis, Banker, Charnes e Cooper (1984) apresentam um modelo que inclui retornos variáveis de escala, o que forneceu a possibilidade de comparar unidades tomadoras de decisões com portes diferentes, chamado de BCC. A fronteira de eficiência do modelo de retornos variáveis de escala (RVE) é representado pela linha $\mathrm{FE}_{\mathrm{RVE}}$. Nele os pontos A, B, C, D e E são considerados eficientes por estarem na fronteira de produção. As DMU's F e G indicam ineficiência (MATTOS; TERRA, 2015b).

Os modelos CCR e BCC podem trazer resultados divergentes pois uma DMU por ser eficiente pelo BCC mas não pelo CCR. Isso ocorre devido o modelo CCR calcular a eficiência total, enquanto o modelo BCC calcula a eficiência técnica. Como Boueri (2015) apresenta, a ineficiência de determinada DMU pode ser estimada pela magnitude da projeção necessária para que ela alcance a referida fronteira (alvo). Essa projeção, que pode ser uma contração dos insumos ou uma expansão dos produtos, é realizada de forma proporcional. Por esse motivo é denominada projeção radial, pois sempre pode ser expressa como um raio partindo da origem, como pode ser vista na Figura 1, nos pontos G-Ga para o modelo BCC e G-Gb no modelo CCR.

Vale mencionar que as unidades tomadoras de decisões (DMU's) podem estar operando com folgas, utilizando insumos além do estritamente necessário. Na DEA a folga é expressa pelo movimento paralelo 
aos eixos das coordenadas da DMU ineficiente, sempre que unidades produtoras usem recursos desnecessários ou aquém do recomendável.

Esse movimento de folga pode ser compreendido no trecho F-Fa, em que, por eliminação dos desperdícios a DMU consegue melhorar sua eficiência, pois produz o mesmo, com menos. Assim, com a eliminação das folgas uma DMU consegue diminuir o movimento radial para alcance do alvo (fronteira de eficiência) (FERREIRA; GOMES, 2012; BOUERI, 2015; MATTOS; TERRA, 2015b; PASCOTTO; COMUNELO; CERETTA, 2017), conforme Quadro 1.

\section{Quadro 1 - Categorias para Análise de Eficiência da DMU}

\begin{tabular}{|c|c|c|}
\hline Categoria & Eficiente & Ineficiente \\
\hline Constante & $\begin{array}{l}\text { Esta é a melhor situação. A DMU utiliza os } \\
\text { recursos sem desperdício e opera com escala } \\
\text { ótima. O aumento da produção deve ocorrer } \\
\text { mantendo-se a proporção de uso dos fatores. } \\
\text { Os aumentos de custos são proporcionais } \\
\text { aos aumentos de produção. }\end{array}$ & $\begin{array}{l}\text { Apesar de estar operando em escala ótima, existe } \\
\text { ineficiência técnica. Isso significa que se pode } \\
\text { reduzir o uso dos insumos e continuar } \\
\text { produzindo a mesma quantidade (orientação } \\
\text { insumo). De maneira equivalente, a produção } \\
\text { pode crescer utilizando-se os mesmos insumos } \\
\text { (orientação produto). Ao eliminar as } \\
\text { ineficiências técnicas, a DMU torna-se eficiente } \\
\text { com retornos constantes. }\end{array}$ \\
\hline Crescente & $\begin{array}{l}\text { Apesar de tecnicamente eficiente, não } \\
\text { existem insumos utilizados em excesso e o } \\
\text { volume de produção está abaixo da escala } \\
\text { ótima. Isso significa que a DMU pode } \\
\text { aumentar a produção a custos decrescentes. } \\
\text { Assim, o aumento da produção deve ocorrer } \\
\text { mediante incorporação de insumos, porém } \\
\text { mantendo-se as relações entre as } \\
\text { quantidades de produtos e insumos. }\end{array}$ & $\begin{array}{l}\text { Nessa situação, existem dois problemas: } \\
\text { ineficiência técnica devido ao uso excessivo de } \\
\text { insumos, e ineficiência de escala. Essa última } \\
\text { ocorre, pois, a DMU está operando abaixo da } \\
\text { escala ótima. Para aumentar a eficiência técnica } \\
\text { devem-se eliminar os excessos de uso dos } \\
\text { insumos. Para operar em escala ótima é } \\
\text { necessário aumentar a produção. Em síntese, a } \\
\text { DMU deve aumentar a produção. Porém esse } \\
\text { aumento deve ocorrer, a fím de que as relações } \\
\text { entre quantidades utilizadas de insumo e o } \\
\text { volume de produção sejam reduzidas. }\end{array}$ \\
\hline Decrescente & $\begin{array}{l}\text { DMU tecnicamente eficiente, porém } \\
\text { operando acima da escala ótima. Uma } \\
\text { alternativa é reduzir o volume de produção } \\
\text { da DMU, mantendo-se a mesma relação } \\
\text { entre insumos e produtos. Uma vez que não } \\
\text { há ineficiência técnica, a super utilização da } \\
\text { planta poderá ser vantajosa. Outra } \\
\text { alternativa para aumentar a produção seria a } \\
\text { adoção de políticas quantitativas, ou seja, o } \\
\text { aumento da produtividade dos fatores } \\
\text { possibilitaria o crescimento da produção } \\
\text { sem necessidade de se utilizarem mais } \\
\text { insumos. O fato é que, nessa situação, o } \\
\text { aumento da produção dar-se-á a custos } \\
\text { crescentes. }\end{array}$ & $\begin{array}{l}\text { Nesse caso a DMU está operando acima da } \\
\text { escala ótima e tem ineficiência técnica. É preciso } \\
\text { corrigir os dois problemas. Para aumentar a } \\
\text { eficiência técnica, devem-se eliminar os } \\
\text { excessos de utilização dos insumos, o que } \\
\text { equivale a produzir mais e com os mesmos } \\
\text { insumos. Com relação à escala, pode-se reduzir a } \\
\text { produção em cada DMU ou utilizar um número } \\
\text { maior de DMU's menores para produzir a mesma } \\
\text { quantidade anterior. Isso dependerá das } \\
\text { condições objetivas de mercado, da } \\
\text { competitividade e estrutura do setor. Pode-se, } \\
\text { ainda, melhorar a tecnologia, aumentando a } \\
\text { produtividade dos fatores de produção ou } \\
\text { insumos. }\end{array}$ \\
\hline
\end{tabular}

Fonte: Adaptado de Ferreira e Gomes (2012, p. 202). 
Para a identificação do tipo de rendimento foi utilizado o método Seiford-Zhu, onde DMU's com eficiência de escala igual a 1 operam com rendimentos constante de escala. Em contrapartida, as DMU's que possuem um valor do somatório dos vetores lambdas $(\lambda)$ ótimo calculados com rendimentos constantes de escala (RCE) menor que um, possui um rendimento crescente de escala, e se este for maior que um, tem-se um rendimento decrescente de escala (COOPER et al., 2006; FERREIRA; GOMES, 2012).

Seguindo Boueri (2015, p. 285) os vetores apresentam os pesos para fazer as melhores combinações lineares com os vetores de insumos e de produtos de todas as DMUs. Este procedimento pode ser expresso pela seguinte notação matricial:

$$
\left\{\begin{array}{c}
\operatorname{Min}_{(\theta, \lambda)}=\theta_{i} \\
\text { s.t. }: \theta_{i} \overline{x_{l}}-X \bar{\lambda} \geq \overline{0_{m}} \\
Q \bar{\lambda}-\overline{q_{l}} \geq \overline{0_{s}} \\
\bar{\lambda} \geq \overline{0_{n}}
\end{array}\right.
$$

Onde $\theta_{i}$ é o escore de eficiência da DMU, $\bar{x}_{l}$ é o vetor de insumos utilizados pela DMU, $\overline{q_{l}}$ é o vetor de produtos gerados, $\bar{\lambda}$ é o vetor de pesos para as combinações lineares, $X$ é uma matriz formada pelos vetores de insumos transpostos de todas as DMUs da amostra, $Q$ é uma matriz formada pelos vetores transpostos de produtos de todas as DMUs e $\theta_{i}$ é o vetor nulo com a dimensão $j$ apropriada. Para este cálculo, utilizou-se, tal como mencionado anteriormente, o Software R, especificamente o pacote Benchmarking.

A DEA foi utilizada para a análise e comparativo da eficiência dos municípios paranaenses por se caracterizar como uma técnica estruturada que permite comparar unidades tomadoras de decisões com portes diferentes, com uso de múltiplos inputs e outputs. Como resultados o modelo permite separar as organizações eficientes das ineficientes, indica a eficiência de cada unidade, fornece indicativos para que cada unidade se torne eficiente, indica uma unidade espelho eficiente para cada unidade ineficiente e mostra a contribuição de cada input e output para a eficiência. 
GRESELE, W. D.; CUNICO, E. A eficiência dos gastos municipais em educação...

\section{Resultados E Discussão}

\subsection{Análise Descritiva}

A Tabela 1 apresenta estatísticas descritivas dos inputs investimentos por aluno, número de docentes por aluno e número de escolas por 100 alunos e o output IDEB.

Tabela 1 - Resumo das estatísticas descritivas dos inputs e outputs.

\begin{tabular}{lcccc}
\hline Estatística descritiva & Investimento por aluno & Docentes por aluno & $\begin{array}{c}\text { Escolas } \\
\text { por 100 alunos }\end{array}$ & IDEB \\
\hline Média & $5.829,98$ & 0,1880 & 0,8579 & 6,14 \\
Mediana & $5.594,04$ & 0,1800 & 0,6800 & 6,10 \\
Desvio & $1.717,93$ & 0,0637 & 0,5990 & 0,67 \\
Coef. de dispersão & 0,29 & 0,3375 & 0,6981 & 0,11 \\
Maior & $11.603,68$ & 0,6500 & 5,9600 & 8,70 \\
Menor & 822,02 & 0,0500 & 0,2500 & 4,30 \\
$1^{\circ} \mathrm{Q}$ & $4.579,44$ & 0,1500 & 0,5200 & 5,70 \\
$3^{\circ} \mathrm{Q}$ & $6.832,50$ & 0,2200 & 0,9700 & 6,60 \\
\hline
\end{tabular}

Fonte: Dados da pesquisa.

Quanto ao resultado do IDEB, os municípios alcançaram uma média de 6,14, com desvio padrão de 0,67 e 0,11 de coeficiente de dispersão, que indica a menor dispersão de dados das variáveis estudadas. Os municipios que tiveram as maiores notas foram: Serranópolis do Iguaçu $(8,7)$; Sertaneja $(8,2)$; Pitangueiras $(7,8)$; Nova Londrina $(7,8)$; Joaquim Távora $(7,7)$; Floraí $(7,7)$ e Bom Sucesso do Sul $(7,7)$. Em contraste, Reserva do Iguaçu $(4,8)$; Foz do Jordão $(4,8)$; Tunas do Paraná $(4,8)$; Guaraqueçaba $(4,8)$; Santa Maria do Oeste $(4,7)$; Goioxim $(4,6)$ e Lupionópolis $(4,3)$ obtiveram os piores resultados.

Na Figura 2 pode-se visualizar as escalas de notas alcançadas pelos municípios. A escala foi desenvolvida conforme o critério de quebras naturais (Jenks), desenvolvidas no software QGis, com pontos de quebra que melhor agrupem valores similares e maximizem a diferença entre as classes. 


\section{Figura 2 - Distribuição espacial dos Índices de Desenvolvimento da Educação Básica dos Municipios do Paraná}

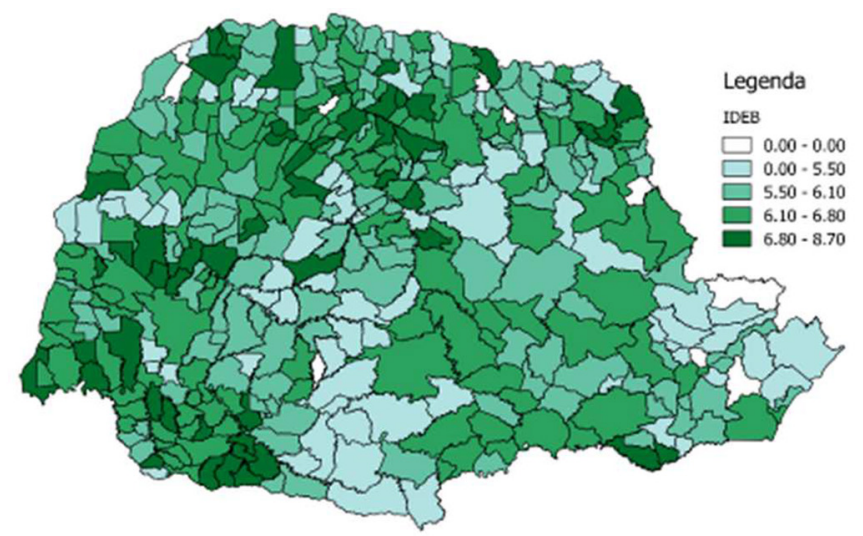

Fonte: Elaboração própria.

Quanto ao input investimento por aluno, apreende-se uma média de $R \$ 5.829,98$, com desvio padrão de cerca de $R \$ 1.717,93$, dado um coeficiente de variação de 0,29 . Os municípios que mais aportaram recursos na educação (em reais $\mathrm{R} \$$ ) foram Campina do Simão $(10.522,87$ ); Guaporema $(10.583,18)$; Nova Aliança do Ivaí $(10.758,14)$; Miraselva $(11.022,62)$; Itaipulândia $(11.449,94)$; Alto Paraíso $(11.498,00)$; Porto Barreiro $(11.603,68)$.

Em contrapartida os municípios que menos investiram (em reais $\mathrm{R} \$$ ) foram: Boa Esperança do Iguaçu $(822,02)$; Moreira Sales $(2.126,11)$; Lapa $(2.600,16)$; Cambé $(2.734,55)$; Sarandi $(2.747,38)$; Jacarezinho $(2.966,98)$ e Paiçandu $(3.005,38)$. Na Figura 3 pode-se visualizar a distribuição espacial dos municipios e a escala do input investimento por aluno, desenvolvida conforme o critério de quebras naturais (Jenks). 
Figura 3 - Distribuição espacial dos investimentos por aluno em Educação Básica dos Municipios do Paraná

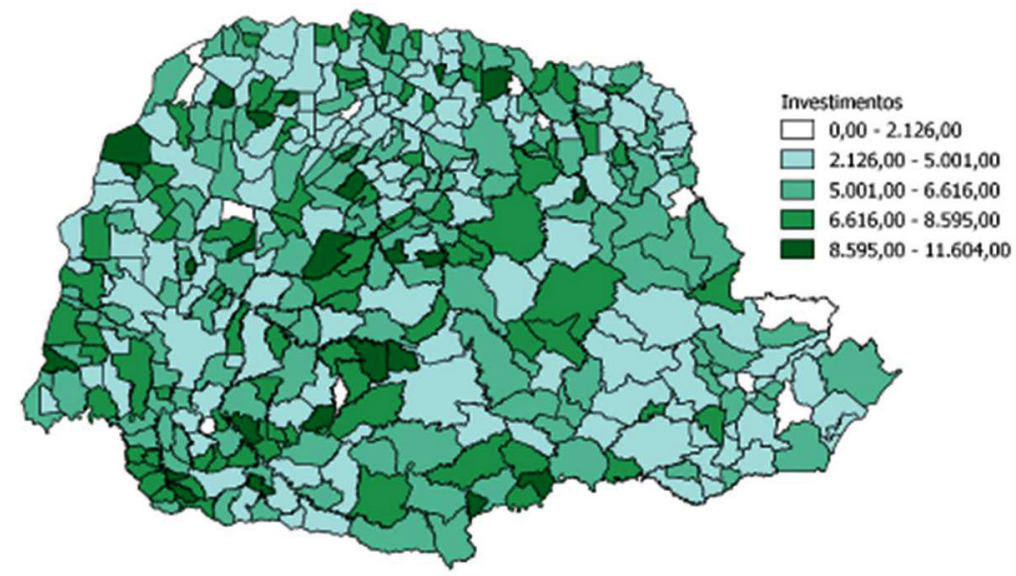

Fonte: Elaboração própria.

A média de docentes por aluno foi de 0,188; com um desvio padrão de 0,0637 e coeficiente de variação de 0,3375 . Os municípios com a maior quantidade de alunos por professor foram Carlópolis (0,052822); Telêmaco Borba $(0,054254)$; Antonina (0,054729); Bocaiúva do Sul (0,065846); Cerro Azul (0,067657); Tibagi (0,071145); e Jaguapitã $(0,076102)$. Os municípios com a menor quantidade de alunos por professor foram Wenceslau Braz (0,307933); Entre Rios do Oeste (0,345455); Rio Bom (0,369565); Iracema do Oeste $(0,388)$; Mercedes (0,488127); Vitorino (0,511078); Nova Santa Bárbara $(0,646421)$.

O input número de escolas por 100 alunos demonstra uma média 0,8579; desvio padrão de 0,5990 e coeficiente de variação de 0,6981. Deve-se ater, tal como ocorre no input anterior, um índice menor indica uma quantidade maior de alunos por escola e, logicamente, um número maior indica uma quantidade menor de alunos por escola. Os municípios Doutor Ulysses $(5,96$; Guaraqueçaba $(4,22)$; Altamira do Paraná $(3,90)$; Cândido de Abreu (3,71); Adrianópolis (3,50); São Jerônimo da Serra (3,40); e Cruz Machado (2,71) apresentaram os menores números de alunos por e escola. Já Cafelândia $(0,31)$; Londrina $(0,31)$; Bom Sucesso (0,30); Paraíso do Norte $(0,28)$; Fazenda Rio Grande $(0,27)$; São Carlos do Ivaí $(0,25)$; Renascença $(0,25)$ apresentaram as maiores quantidades de alunos por escolas, conforme a Figura 4. 
Figura 4 - Distribuição espacial dos números de escolas por 100 alunos na Educação Básica dos Municípios do Paraná

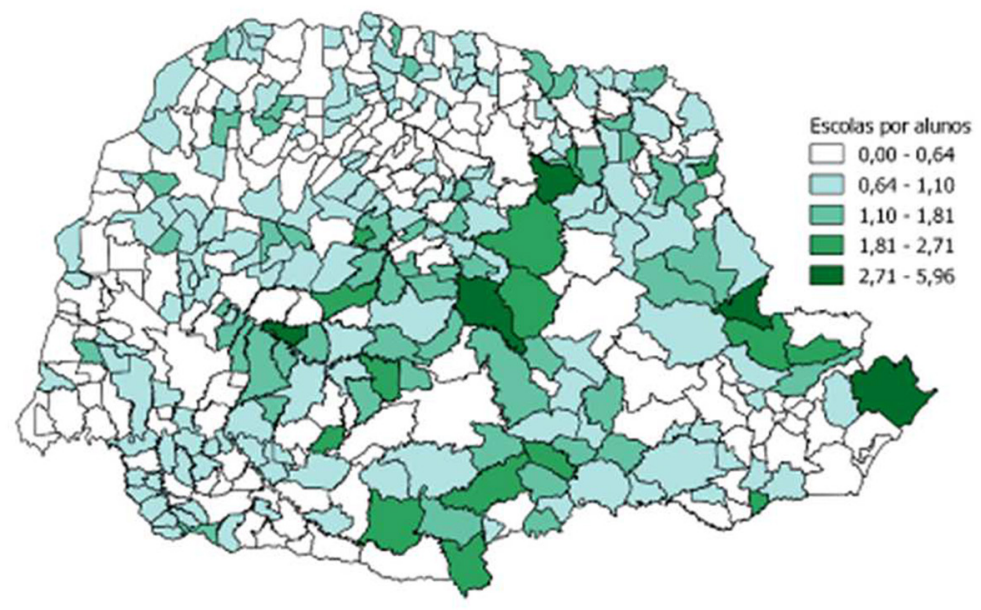

Fonte: Elaboração própria.

\subsection{Análise Envoltória de Dados}

Inicialmente foi utilizado o modelo DEA com base nos pressupostos dos retornos constantes de escala (CCR), a fim se se obter uma medida de eficiência técnica (ETccr) para cada município em estudo. Após, o fundamento de retornos constantes foi retirado, adicionando uma restrição de convexidade, que permitiu a obtenção de medidas de eficiência técnica com base em retornos variáveis (ETbcc). De posse das medidas de eficiência técnica com retornos variáveis e constantes, foi possivel identificar a eficiência de escala (EE).

A Tabela 2 apresenta estatísticas descritivas dos dados da eficiência técnica (ET) com retornos constantes de escala (CCR), rendimentos variáveis de escala (BCC), eficiência de escala $(\mathrm{EE})$ e uma síntese dos resultados por município e por faixa de eficiência alcançada. 
GRESELE, W. D.; CUNICO, E. A eficiência dos gastos municipais em educação...

Tabela 2 - Resumo da estatística descritiva das eficiências dos Municipios do Paraná

\begin{tabular}{|c|c|c|c|c|c|c|}
\hline \multirow{2}{*}{$\begin{array}{l}\text { Estatística descritiva } \\
\text { Eficiência }<0,1\end{array}$} & \multicolumn{2}{|c|}{ ET(ccr) } & \multicolumn{2}{|c|}{ ET(bec) } & \multicolumn{2}{|c|}{ EE } \\
\hline & 0 & $0,0 \%$ & 0 & $0,0 \%$ & 0 & $0,0 \%$ \\
\hline $0,1 \leq$ Eficiência $<0,2$ & 0 & $0,0 \%$ & 0 & $0,0 \%$ & 0 & $0,0 \%$ \\
\hline $0,2 \leq$ Eficiência $<0,3$ & 8 & $2,1 \%$ & 2 & $0,5 \%$ & 0 & $0,0 \%$ \\
\hline $0,3 \leq$ Eficiência $<0,4$ & 56 & $14,4 \%$ & 26 & $6,7 \%$ & 0 & $0,0 \%$ \\
\hline $0,4 \leq$ Eficiência $<0,5$ & 70 & $18,0 \%$ & 74 & $19,0 \%$ & 0 & $0,0 \%$ \\
\hline $0,5 \leq$ Eficiência $<0,6$ & 89 & $22,9 \%$ & 86 & $22,1 \%$ & 0 & $0,0 \%$ \\
\hline $0,6 \leq$ Eficiência $<0,7$ & 76 & $19,5 \%$ & 83 & $21,3 \%$ & 2 & $0,5 \%$ \\
\hline $0,7 \leq$ Eficiência $<0,8$ & 43 & $11,1 \%$ & 49 & $12,6 \%$ & 20 & $5,1 \%$ \\
\hline $0,8 \leq$ Eficiência $<0,9$ & 21 & $5,4 \%$ & 35 & $9,0 \%$ & 97 & $24,9 \%$ \\
\hline $0,9 \leq$ Eficiência $<1,0$ & 15 & $3,9 \%$ & 14 & $3,6 \%$ & 259 & $66,6 \%$ \\
\hline Eficiência $=1,0$ & 11 & $2,8 \%$ & 20 & $5,1 \%$ & 11 & $2,8 \%$ \\
\hline Média & \multicolumn{2}{|c|}{$58,5 \%$} & \multicolumn{2}{|c|}{$62,8 \%$} & \multicolumn{2}{|c|}{$92,8 \%$} \\
\hline Mediana & \multicolumn{2}{|c|}{$56,9 \%$} & \multicolumn{2}{|c|}{$61,1 \%$} & \multicolumn{2}{|c|}{$\begin{array}{c}95,1 \% \\
6.9 \%\end{array}$} \\
\hline Desvio & & & \multirow{2}{*}{\multicolumn{2}{|c|}{$\begin{array}{l}17,3 \% \\
27,6 \%\end{array}$}} & & \\
\hline Coef.de variação & \multicolumn{2}{|c|}{$30,0 \%$} & & & \multicolumn{2}{|c|}{$7,5 \%$} \\
\hline Mínimo & \multicolumn{2}{|c|}{$25,6 \%$} & \multicolumn{2}{|c|}{$27,9 \%$} & \multicolumn{2}{|c|}{$67,3 \%$} \\
\hline $1^{\circ}$ Quartil & \multicolumn{2}{|c|}{$44,8 \%$} & \multicolumn{2}{|c|}{$49,4 \%$} & \multicolumn{2}{|c|}{$88,6 \%$} \\
\hline $3^{\circ}$ Quartil & \multicolumn{2}{|c|}{$68,4 \%$} & \multicolumn{2}{|c|}{$74,2 \%$} & \multicolumn{2}{|c|}{$98,6 \%$} \\
\hline
\end{tabular}

Fonte: Elaboração própria.

Os municípios de Altônia, Boa Esperança do Iguaçu, Cafelândia, Cambé, Carlópolis, Japurá, Jussara, Paiçandu, Renascença, Serranópolis do Iguaçu e Telêmaco Borba, se destacam por possuírem eficiência técnica total (ETccr), com retornos constantes de escala. O nível médio de eficiência técnica é de $58,5 \%$, com um desvio padrão de 17,5 . Isso significa que os municípios podem, em média, reduzir $41,5 \%$ dos insumos sem comprometer seus resultados. Os produtores que alcançaram a máxima eficiência técnica não podem reduzir os gastos sem comprometer resultados, mas os demais o podem, tendo como referência aqueles que tiveram uma eficiência técnica (ETccr) igual a um.

Quando se assume o pressuposto de retornos constantes de escala as fontes de ineficiência podem incluir as decorrentes da incorreta escala de produção, sendo então necessário identificar a eficiência técnica com retornos variáveis de escala. No critério de Eficiência Técnica (ETbcc), ou seja, a eficiência com retornos variáveis de escala, a média de eficiência foi de $62,8 \%$, com desvio-padrão de 17,3. Em destaque, os municípios Altônia, Boa Esperança do Iguaçu, Cafelândia, Cambé, Carlópolis, Japurá, Jussara, Paiçandu, Renascença, Serranópolis do Iguaçu, Telêmaco Borba, Maringá, Paraíso do Norte, São Carlos do Ivaí, Atalaia, Medianeira, Sarandi, Moreira Sales e Tibagi, foram considerados eficientes. Essa condição reforça os achados de Zubyk et al. (2019) uma vez que ao analisar as decisões e comportamentos, é possivel maximizar eficiência na gestão pública. 
Sabe-se que uma condição para que uma DMU apresente a máxima eficiência técnica com retornos constantes é que sua eficiência com retornos variáveis também seja máxima (FERREIRA; GOMES, 2012). Conclui-se que dos 20 municípios eficientes no modelo de retornos variáveis, 11 são igualmente eficientes com retornos constantes.

Um ponto que emerge na comparação dos modelos de retornos constantes (CCR) e retornos variáveis (BCC), está na possibilidade de decomposição da eficiência relativa da DMU em eficiência técnica e eficiência de escala, sendo possivel avaliar quanto da ineficiência da DMU é devida a sua incapacidade técnica e quanto é devida ao fato dela não produzir na escala apropriada (FERREIRA; GOMES, 2012).

A medida de eficiência de escala, é obtida pela razão entre as medidas de eficiência técnica dos modelos com retornos constantes e com retornos variáveis. Se o resultado for igual a um (1), indica que o município está operando em escala ótima. Assim, quanto à eficiência de escala (EE), 11 municipios foram considerados eficientes, pois estavam operando nas fronteiras de eficiência, conforme ponto $\mathrm{C}$ da Figura 01.

Nas análises das eficiências total, técnica e de escala, percebe-se não haver indícios de problema quanto à eficiência de escala, pois há média alta de cerca de $93 \%$ e baixo desvio padrão. Como a eficiência total (ETccr) é o produto da eficiência de escala (EE) pela eficiência técnica (ETbcc), pode-se concluir que o principal problema dos municípios está ligado à eficiência técnica, conforme Figura 5.

Figura 5 - Distribuição Espacial das Eficiências de Escala dos Municipios do Paraná

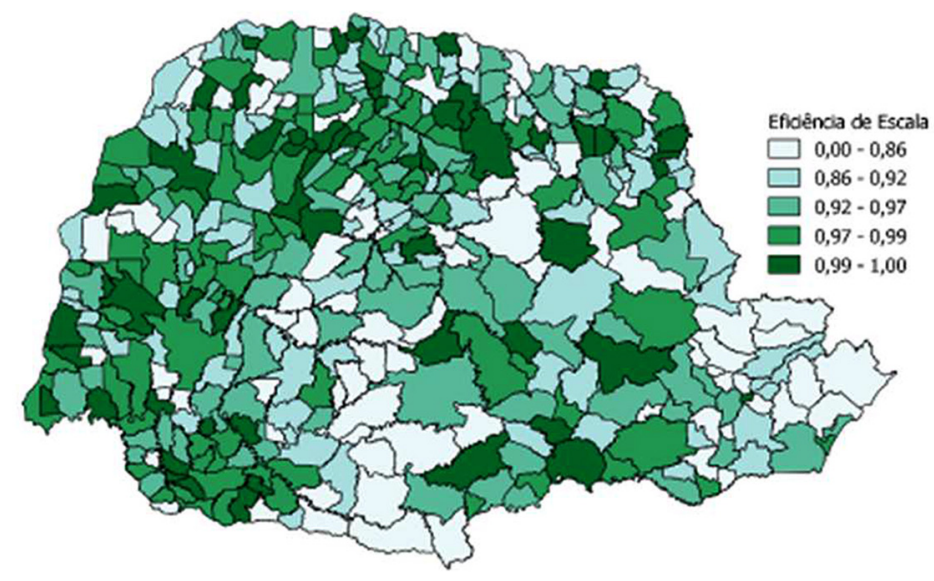

Fonte: Elaboração própria. 
É reconhecido na literatura sobre a DEA que as DMU's podem estar operando em um dos três diferentes tipos de rendimentos. Para identificação do tipo de rendimento, pode-se utilizar o método SeifordZhu, onde DMU's com eficiência de escala igual a 1 operam com rendimentos constante de escala. Em contrapartida, as DMU's que possuem um valor do somatório dos lambdas ótimo calculados com rendimentos constantes de escala ( $\mathrm{RCE}$ ) menor que um, possui um rendimento crescente de escala, e se este for maior que um, tem-se um rendimento decrescente de escala (COOPER et al., 2006). Foi constatado que 278 municípios possuíram retornos de escala crescente, 100 municípios retornos de escala decrescente e 11 com retornos de escala constante, conforme Figura 6.

\section{Figura 6 - Distribuição Espacial dos Tipos de Rendimentos dos Municipios do}

Paraná

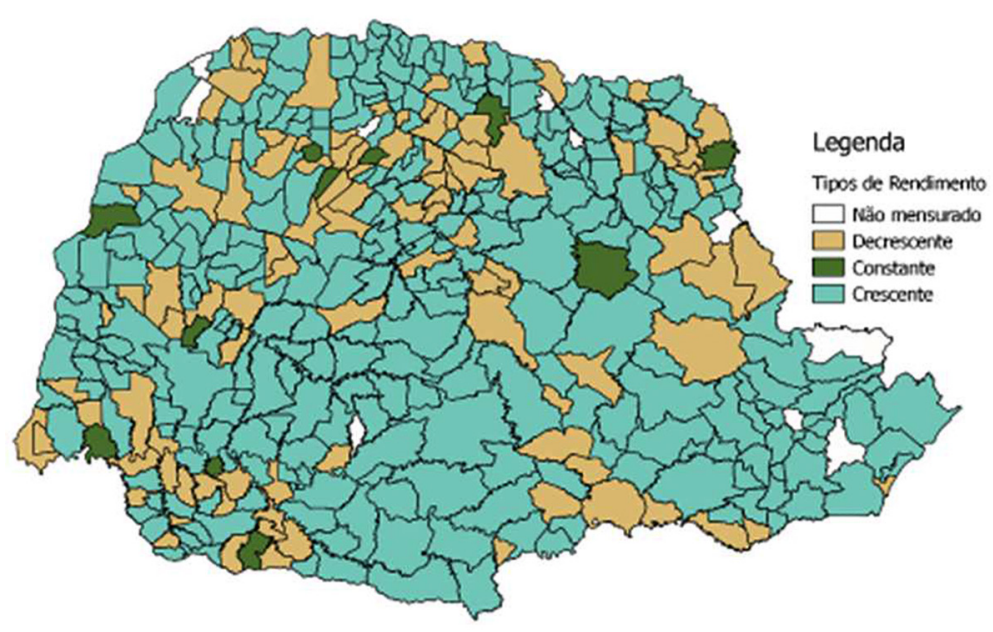

Fonte: Elaboração própria.

Conforme delineado no quadro teórico, após a análise do modelo DEA as DMU's podem ser classificadas segundo duas categorias: tecnicamente eficientes ou ineficientes. E, dessa forma, podem estar operando com retornos constantes, crescentes ou decrescentes. Esta classificação das DMU's com eficiência técnica ou ineficiência técnica e tipos de retornos de escala, possibilita o enquadramento dos municípios em seis tipificações, conforme delineado no quadro teórico (FERREIRA; 
GOMES, 2012). Os dados da Tabela 3, fornecem um detalhamento dos municípios segundo o tipo de retorno (crescente, constante e decrescente), se é eficiente ou ineficiente e informações sobre as médias das eficiências (constante, variável e de escala), as médias dos recursos aportados (Inputs) e o resultado médio (Output), individualmente.

Tabela 3 - Perfil médio de eficiência dos municipios do Paraná no ano de 2017

\begin{tabular}{l|c|c|c|c}
\hline \multicolumn{1}{c}{ Especificação } & \multicolumn{3}{c|}{ Tipo de retorno } & \multirow{2}{*}{ Média } \\
\cline { 2 - 4 } & Crescente & Constante & Decrescente & - \\
\hline Eficientes & 6 & 11 & 3 & - \\
Ineficientes & 272 & 0 & 97 & 0,927725687 \\
Eficiência Total (ETccr) & 0,91000461 & 1 & 0,969040107 & 0,627558642 \\
Eficiência Técnica (ETbcc) & 0,599436463 & 1 & 0,664769752 & 0,584522632 \\
Eficiência de Escala (EE) & 0,546934856 & 1 & 0,643314138 & 0,188817481 \\
Docentes por aluno & 0,187841727 & 0,141818182 & 0,1967 & 5829,98 \\
Investimento por aluno & $5.972,58$ & $3.609,26$ & $5.677,80$ & 0,8579434445 \\
Escolas por 100 alunos & 0,895683453 & 0,453636364 & 0,7975 & 6,143958869 \\
IDEB & 5,836690647 & 6,890909091 & 6,916 & \\
\hline
\end{tabular}

Fonte: Elaboração própria.

Dos 389 municipios paranaenses analisados, 272 (70\%), são ineficientes e possuem retornos de escala crescente; 97 (25\%) municípios são ineficientes e possuem retornos decrescentes de escala; 20 (5\%) são eficientes (RVE) sendo 11 com retornos constante, 6 crescentes e 3 decrescente. Não foram identificados municípios com ineficiência técnica e retorno de escala constante, conforme Figura 7.

\section{Figura 7 - Distribuição Espacial das Tipificações das Eficiências dos Municipios do Paraná}

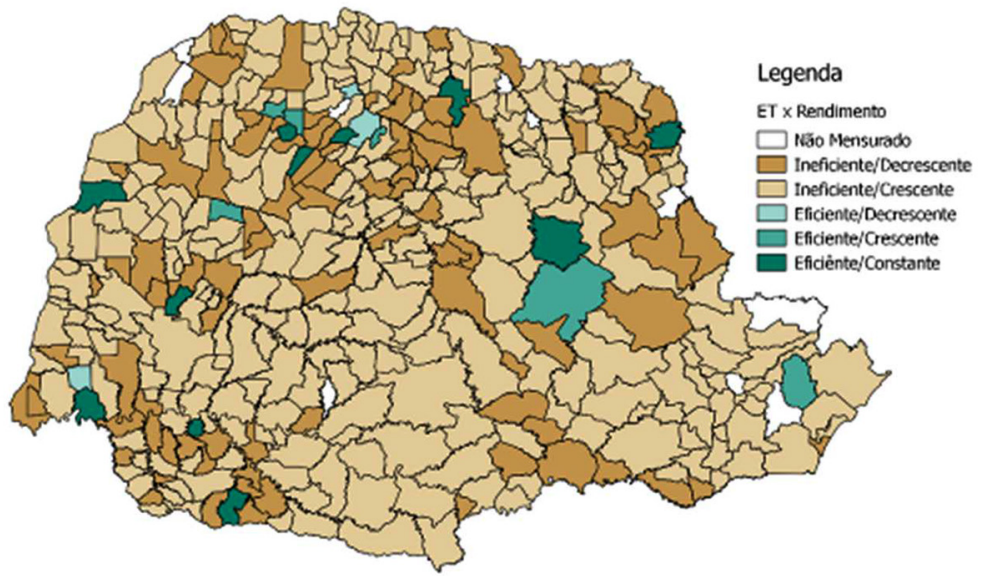

Fonte: Elaboração própria. 
GRESELE, W. D.; CUNICO, E. A eficiência dos gastos municipais em educação...

A maioria dos municípios foram enquadrados com status de ineficiência técnica com retorno de escala crescente. Nesse cenário, eles estavam operando abaixo da escala ótima (precisam melhorar o resultado), além de apresentarem ineficiência técnica (há desperdício de recursos).

Comparando as medidas de eficiência média, percebe-se diferenças entre os problemas encontrados nos municipios ineficientes. Os que possuem retornos crescentes, o uso de insumos é maior quanto aos fatores "investimento por aluno" e "escolas por 100 alunos", em comparação aos municipios que apresentam escalas decrescentes, ou seja, mesmo esses municípios aportando mais recursos, não conseguem ter melhores resultados. Do total, 97 municipios possuem ineficiência técnica com retorno de escala decrescente. Estão acima da escala ótima e apresentam desperdícios, necessitando reduzir utilização dos recursos.

Foram encontrados 11 municípios com eficiência técnica com retorno de escala constante, sendo esses: Altônia; Boa Esperança do Iguaçu; Cafelândia; Cambé; Carlópolis; Japurá; Jussara; Paiçandu; Renascença; Serranópolis do Iguaçu e Telêmaco Borba. Estes, operam na melhor situação, sendo assim o aumento da produção deve ocorrer mantendo-se a proporção de uso dos fatores e os aumentos de custos são proporcionais aos aumentos de produção. Dos municípios paranaenses, seis possuem eficiência técnica com retorno de escala crescente, em que a DMU está operando sem desperdício de recursos. No entanto, estão abaixo da escala ótima, assim, um aumento da produção pode ocorrer através da incorporação de insumos, desde que mantida a relação entre quantidades de resultados x insumos utilizados.

Apenas 3 municipios possuem eficiência técnica com retorno de escala decrescente. Estes operam sem desperdícios, mas acima da escala ótima, necessitando a adoção de políticas quantitativas que visem o aumento da produção sem utilizar mais recursos, evitando custos crescentes.

A projeção necessária para uma DMU alcançar a fronteira sempre tem como referência outros pontos, ou seja, os pontos que dão origem ao trecho da fronteira sobre o qual a projeção de um determinado ponto recai. Esses formam o conjunto de referência da DMU em questão 
(benchmarks). Para identificá-las, deve-se encontrar os valores calculados para o vetor $\chi$ (lambda) (BOUERI, 2015). Na análise, os municipios que são referências para outros municípios foram Paiçandu (benchmark para 101 municípios); Sarandi (88); Telêmaco Borba (65); Tibagi (56); Boa Esperança do Iguaçu (30); Moreira Sales (17); São Carlos do Ivaí (11); Jussara (10); Altônia (3). Os municípios eficientes Carlópolis (1); Cafelândia (1); Japurá (1); Medianeira (1); Serranópolis do Iguaçu (1) não são referências para outros municípios, pois o município eficiente o possui como autorreferência.

Como contribuição teórica, os resultados do Quadro 2, relacionam as medidas de desempenho com os indicadores analisados, permitindo análise sintética da evolução dos resultados periodicamente comparados.

\section{Quadro 2 - Métrica para análise de eficiência individual do municipio}

\begin{tabular}{|c|c|c|c|c|}
\hline & $\begin{array}{l}\text { INVESTIMENTOS/ } \\
\text { MATRÍCULA }\end{array}$ & $\begin{array}{l}\text { DOCENTES/ } \\
\text { MATRÍCULA }\end{array}$ & $\begin{array}{c}\text { ESCOLAS/ } \\
\text { MATRÍCULA }\end{array}$ & IDEB 2017 \\
\hline Ineficiente/Decrescente & $\begin{array}{l}\text { Abaixo da Média: } 37 \\
\text { casos acima da média } \\
\text { e } 60 \text { abaixo da média }\end{array}$ & $\begin{array}{l}\text { Acima da Média: } \\
50 \text { casos acima da } \\
\text { média e } 47 \text { abaixo } \\
\text { da média }\end{array}$ & $\begin{array}{l}\text { Abaixo da Média: } \\
31 \text { casos acima da } \\
\text { média e } 66 \text { abaixo } \\
\text { da média }\end{array}$ & $\begin{array}{l}\text { Acima da Média: } \\
97 \text { casos acima da } \\
\text { média }\end{array}$ \\
\hline Ineficiente/Crescente & $\begin{array}{l}\text { Acima da Média: } 141 \\
\text { casos acima da média } \\
\text { e } 131 \text { abaixo da média }\end{array}$ & $\begin{array}{l}\text { Acima da Média: } \\
127 \text { casos acima } \\
\text { da média e } 145 \\
\text { abaixo da média }\end{array}$ & $\begin{array}{l}\text { Acima da Média: } \\
102 \text { casos acima } \\
\text { da média e } 170 \\
\text { abaixo da média }\end{array}$ & $\begin{array}{l}\text { Abaixo da Média: } \\
77 \text { casos acima da } \\
\text { média e } 195 \\
\text { abaixo da média }\end{array}$ \\
\hline Eficiente/Decrescente & $\begin{array}{l}\text { Abaixo da Média: } 03 \\
\text { casos abaixo da média }\end{array}$ & $\begin{array}{l}\text { Abaixo da Média: } \\
2 \text { casos acima da } \\
\text { média e } 1 \text { abaixo } \\
\text { da média }\end{array}$ & $\begin{array}{c}\text { Abaixo da Média: } \\
03 \text { casos abaixo } \\
\text { da média }\end{array}$ & $\begin{array}{c}\text { Acima da Média: } \\
03 \text { acima da } \\
\text { média }\end{array}$ \\
\hline Eficiente/Crescente & $\begin{array}{l}\text { Abaixo da Média: } 1 \\
\text { caso acima da média e } \\
5 \text { abaixo da média }\end{array}$ & $\begin{array}{c}\text { Abaixo da Média: } \\
1 \text { caso acima da } \\
\text { média e } 5 \text { abaixo } \\
\text { da média }\end{array}$ & $\begin{array}{c}\text { Abaixo da Média: } \\
6 \text { casos abaixo da } \\
\text { média }\end{array}$ & $\begin{array}{c}\text { Abaixo da Média: } \\
01 \text { casos acima da } \\
\text { média e } 5 \text { abaixo } \\
\text { da média }\end{array}$ \\
\hline Eficiente/Constante & $\begin{array}{l}\text { Abaixo da Média: } 01 \\
\text { caso acima da média e } \\
10 \text { abaixo da média }\end{array}$ & $\begin{array}{l}\text { Abaixo da Média: } \\
4 \text { casos acima da } \\
\text { média e } 7 \text { abaixo } \\
\text { da média }\end{array}$ & $\begin{array}{l}\text { Abaixo da Média: } \\
1 \text { caso acima da } \\
\text { média e } 10 \text { abaixo } \\
\text { da média }\end{array}$ & $\begin{array}{c}\text { Acima da Média: } \\
11 \text { casos acima da } \\
\text { média }\end{array}$ \\
\hline
\end{tabular}

Fonte: Elaboração própria.

Ao tratar da eficiência no uso de recursos públicos, constata-se uma melhora no quantitativo de municipios eficientes, se comparado ao estudo de Gresele e Krukoski (2015), no estado do Paraná, em 2017; 20 municípios apresentaram eficiência na gestão dos recursos da edu- 
cação, já em 2015, 13 municípios foram considerados eficientes. Entretanto houve apenas uma pequena melhora na média da eficiência (bcc) dos municípios, de cerca de 60\% de eficiência em 2015 para 62\% no ano de 2017. Apesar de, como pontuam Savian e Bezerra (2013), ter havido uma redução do número de municípios eficientes, entre os anos de 2005 e 2009, e uma melhora até o ano de 217, ainda há um pequeno grupo de municípios no Estado no Paraná são eficientes e com pouca melhora na eficiência média da região estudada.

\section{Considerações Finais}

A escassez de recursos sempre foi um ponto latente na sociedade. Agravado pelas crises, tanto econômica como sanitária, geram maiores demandas sociais dos governos e uma menor arrecadação, haja vista a queda da renda da população. Considera-se que o problema de pesquisa foi respondido, uma vez que o artigo apresentou as análises das eficiências, individual e relativa, dos municípios paranaenses no ano de 2017. Os objetivos secundários foram atingidos dado que as eficiências, total, de escala e técnica, dos municípios foram analisadas por meio da DEA, com base nos investimentos financeiros por alunos, número de docentes por alunos, número de escolas por alunos e no resultado alcançado no IDEB de cada município.

Individualmente, um número de 15 municípios se destaca como referência para outros municípios e, portanto, há nessa condição uma oportunidade de criação de grupos de aprendizado (benchmark) para outros gestores da educação municipal das localidades que apresentaram ineficiência. Há necessidade de formação continuada do gerenciamento de recursos, voltada aos profissionais que, em sua maioria, apenas dominam conhecimentos pedagógicos em suas respectivas áreas de atuação.

O principal problema dos municípios paranaenses na gestão dos recursos educacionais está relacionado a eficiência técnica, ou seja, utilização dos recursos na busca do resultado. Para tanto, apenas aumentar o volume de recursos, não trará melhores resultados. Há que se considerar novas escolhas de gestão. Do total, 272 municípios são 
ineficientes e possuem retornos de escala crescente. E, 97 municipios são ineficientes, e possuem retornos decrescentes. Com a perspectiva de aumento de aporte de recursos no ensino com o "Novo Fundeb", existe uma latente necessidade de correta alocação em face do tipo de eficiência e escala que os municípios se encontram.

Do ponto de vista teórico, a elaboração do Quadro 2 propõe uma métrica de análise facilitada. Para estudos futuros, indica-se a possibilidade de relacionar outros indicadores de avaliação do desempenho dos outputs, análise por meio de séries temporais e dados em painel.

\section{Referências}

BEGNINI, S.; TOSTA, H. T. A. Eficiência dos Gastos Públicos com a Educação Fundamental no Brasil: uma aplicação da análise envoltória de dados (DEA). E\&G Economia e Gestão, Belo Horizonte, v. 17, n. 46, Jan./Abr. 2017. DOI: https://doi.org/10.5752/P.1984-6606. 2017v17n46p43

BOGETOFT, P.; OTTO, L. Benchmarking with DEA. SFA and R. Springer Science, 2011. DOI: 10.1007/978-1-4419-7961-2.

BOHRER, É.; COMUNELO, A. L.; GODARTH, K. A. Eficiência do Gasto Público na Educação: O caso do Sudoeste do Paraná, Brasil. CAP Accounting and Management, v.7(7), p. 209-222, 2013.

BOUERI, R. Modelos não paramétrico: Análise Envoltória de Dados (DEA) In: BOUERI, R.; ROCHA, F.; RODOPOULOS, F. (organizadores) Avaliação da Qualidade do Gasto Público e Mensuração da Eficiência. Brasília: Secretaria do Tesouro Nacional, 2015.

BRASIL. Constituição (1988). Constituição da República Federativa do Brasil. Brasília. Disponível em: http://www.planalto.gov.br/ccivil_03/ Constituicao/Constituicao.htm

BRASIL. Lei $n^{\circ} 14.113$, de 25 de dezembro de 2020. Regulamenta o Fundo de Manutenção e Desenvolvimento da Educação Básica e de Valorização dos Profissionais da Educação (Fundeb). 2020. Disponivel em: http:// www.planalto.gov.br/ccivil_03/_ato2019-2022/2020/lei/L14113.htm. 
BRASIL. Constituição. Emenda constitucional n ${ }^{\circ} 108$, de 26 de agosto de 2020. Altera a Constituição Federal para estabelecer critérios de distribuição da cota municipal e para dispor sobre o Fundo de Manutenção e Desenvolvimento da Educação Básica e de Valorização dos Profissionais da Educação (Fundeb). 1988. Disponivel em: http:/ /www.planalto. gov.br/ccivil_03/Constituicao/Emendas/Emc/emc108.htm.

CASTRO, M. S.; SOUSA, E. P. Eficiência dos Gastos Públicos da Rede de Ensino Municipal Cearense. Gestão \& Regionalidade, v. 34(100), p. 92-109, 2018. DOI: https://doi.org/10.13037/gr.vol34n100.2596

COOPER, W. L.; LAWRENCE, M. S.; TONE, K. Introduction to Data Envelopment Analysis: and its uses with DEA-Solver Software and References. Springer, New York, 2006.

DANCEY, C. P.; REIDY, J. Estatística sem matemática para psicologia. Porto Alegre: Artmed, 2013.

DINIZ, J. A.; CORRAR, L. J. Análise da Relação entre a Eficiência e as Fontes de Recursos dos Gastos Municipais no Ensino Fundamental. Sociedade, Contabilidade e Gestão, v. 6(1), 2011. DOI:https://doi. org/10.21446/scg_ufrj.v6i1.13232

FERREIRA, C. M. de. C., Gomes, A. P. (2012). Introdução à análise envoltória de dados: teoria, modelos e aplicações. Viçosa: Editora UFV.

FIELD, A. Descobrindo a Estatística usando o SPSS. São Paulo: Artmed, 2009.

GRESELE, W. D.; KRUKOSKI, F. A. Eficiência dos Gastos Municipais em Educação no Paraná. Revista Pensamento Contemporâneo em Administração, v. 12(4), p. 56-74, 2018. DOI:http://dx.doi.org/10.12712/ rpca.v12i4.27149.

INEP. Ministério da Educação. Disponivel em: http://www.enem.inep. gov.br/.

LOURENÇO, R. L.; ANGOTTI, M.; NASCIMENTO, J. C. H. B. DO; SAUERBRONN, F. F. Eficiência do gasto público com ensino fundamental: uma análise dos 250 maiores municípios brasileiros. Revista Contabilidade Vista e Revista, v. 28(1), p. 89-116, jan/jun, 2017. 
MATIAS, A. B.; QUAGLIO, G. DE. M.; OLIVEIRA, B. G. DE.; LIMA, J. P. R. DE.; BERTOLIN, R. V. Niveis de Gastos e Eficiência Pública em Educação: Um Estudo de Municípios Paulistas Utilizando Análise Envoltória de Dados. Rev. Adm. UFSM, v. 11(4), p. 1051-1067, 2018. DOI: 10.5902/19834659 16448.

MATTOS, E.; TERRA, R. Conceitos sobre eficiência. In: Boueri, R.; Rocha, F.; Rodopoulos, F. (organizadores) Avaliação da qualidade do gasto público e mensuração da eficiência. Secretaria do Tesouro Nacional: Brasília, (2015a).

MATTOS, E.; TERRA, R. Fundamentos microeconômicos da mensuração da eficiência. In: Boueri, R.; Rocha, F.; Rodopoulos, F. (org.) Avaliação da qualidade do gasto público e mensuração da eficiência. Secretaria do Tesouro Nacional: Brasília, (2015b).

MONTE, M. M.; LEOPOLDINO, C. B. Eficiência dos Gastos Municipais em Educação no Ceará. Revista Pensamento Contemporâneo em Administração, v. 11(4), p. 44-55, 2017. DOI: https://doi.org/10.12712/ rpca.v11i4.1104

PASCOTTO, H.; COMUNELO, A. L.; CERETTA, G. F. Eficiência técnica na aplicação de recursos públicos na área da saúde dos municípios do sudoeste do Estado do paraná. Gestão e Desenvolvimento em Revista, v. 4(1), jan-jun, p. 21-37, 2018. DOI: https://doi.org/10.48075/gd\%20 em\%2Orevista.v4i1.19962.

PEÑA, C. R. Eficiência e impacto do contexto na gestão através do DEA: o caso da UEG. Produção, Brasília, DF, v. 22(4), p. p. 778-787, set./dez, 2012. DOI: http://dx.doi.org/10.1590/S0103-65132012005000086.

ROCHA, F.; DUARTE, J.; GADELHA, S. R. De B.; OLIVEIRA, P. P. De.; PEREIRA, L. F. V. N. É possivel atingir as metas para a educação sem aumentar os gastos? Uma análise para os municípios brasileiros. Textos para Discussão do Tesouro Nacional. nro.15, 2013. Disponivel em: https:/ / publicacoes.tesouro.gov.br/index.php/textos/issue/view/texto 15

SAVIAN, M. P. G.; BEZERRA, F. M. Análise de eficiência dos gastos públicos com educação no ensino fundamental no estado do Paraná. Economia \& Região, v.1 (1), p.26-47, jan./jul, 2013. DOI: http://dx.doi. org/10.5433/2317-627X.2013v1n 1p26 
SENRA, N.; MELLO, S. De.; NANCI, L.C.; MELLO, J. C. C. B. S.; MEZA, L. A. Estudo sobre métodos de seleção de variáveis em DEA. Pesquisa Operacional, v.27(2), Rio de Janeiro May/Aug, 2007. DOI: https://doi. org/10.1590/S0101-74382007000200001.

SILVA, M. Z. DA.; MORETTI, B. R.; SCHUSTER, H. A. Avaliação da eficiência hospitalar por meio da análise envoltória de dados. Revista RGSS, v.5, n. 2, 2016. DOI: http://dx.doi.org/10.5585/rgss.v5i2.248.

SOUSA, A. N. G. De.; RUTALIRA, J. J. B. Eficiência do gasto público em educação de nível fundamental: uma análise dos estados brasileiros. Interface - Revista do Centro de Ciências Sociais Aplicadas, v. 13(1), p. 33-50, 2016.

VIDAL, E. M.; VIEIRA, S. L. Gestão Educacional e Resultados no IDEB: um estudo de caso em dez municípios cearenses. Estudos em Avaliação Educacional, São Paulo, v.22(50), p.419-434, set./dez, 2011.. DOI: https://doi.org/10.18222/eae225020111963

ZUBYK, A. R.; RIBEIRO, F.; CLEMENTE, A.; GERLK, W. Eficiência na gestão dos gastos municipais em educação e de saúde: um estudo baseado na análise envoltória de dados no Estado do Paraná. Ref. Contabilidade, v.38 (1), p. 123-139, jan/abr, 2019. DOI: 10.4025/enfoque. v38i1.39191. 
